\title{
Automatic Extraction of Road Information Using the Object-Oriented Technology
}

\author{
Wang Ping ${ }^{*}{ }^{1,2}$, Zhai Zhaokun ${ }^{1}$, Fu Hui ${ }^{1}$ and Zhao Zhiling ${ }^{1}$ \\ ${ }^{I}$ School of Geomatics, Shandong University of Science and Technology, Qingdao, Shandong, 266590, P.R. China \\ ${ }^{2}$ The Key Laboratory of Marine Surveying and Mapping in Universities of Shandong, 266590, P.R. China
}

\begin{abstract}
Road information is rich in urban districts and mining areas. With the increase in spatial resolution and spectral resolution of remote sensing data, it is possible to extract information of narrow roads. However, the traditional manual extraction method using high-spatial-resolution data has shortcomings of low accuracy and low efficiency. Considering the features of SPOT-5 and Gaofen-1 data and the actual road situation of the study areas, the object-oriented method is used in this paper. The main advantage of this method is overcoming the limit of road extraction only using spectral information. A comparison with the results of the traditional supervised classification based on pixels proves that the objectoriented method improves the accuracy by $10 \%$ and provides better road information results. In addition, it is a more effective method to extract information for geographical condition monitoring.
\end{abstract}

Keywords: Geographical information monitoring, High-resolution image, Object-oriented, Road information extraction.

\section{INTRODUCTION}

In recent years, image resolution has rapidly increased with the development of remote sensing technology. More high-resolution images are used in national geographical information monitoring. Road information is more abundant with the improvement of satellites. It is possible to extract the road information of urban districts and mining areas. The automatic or semi-automatic extraction method has gained increasingly more attention because the traditional manual method has low efficiency and accuracy [1].

Traditional image extraction techniques such as supervised classification and unsupervised classification are pixelbased techniques, which explore the spectral differences of various features to extract the thematic information of interest [2]. Spectral information that is only considered in these methods leads to fragmented and discontinuous road extraction information. The road in mining areas is heterogeneous. Road information is also affected by trees on both sides of the road. One object can have many different spectra, and different objects can have identical spectra [3]. For highresolution images, the extraction method that only uses spectral information cannot satisfy the required efficiency and accuracy. In addition, the methods of artificial neural network and fuzzy classification have difficulties in automatic extraction. Hence, road information is often manually extracted based on visual interpretation and grey pixel. The object-oriented method has been widely examined because the information is extracted based on objects using the spectral, textural and spatial information of a high-resolution image. This method can overcome the problem of salt \&

\footnotetext{
*Address correspondence to this author at the School of Geomatics, Shandong University of Science and Technology, Qingdao, Shandong, 266590, P.R. China; Tel: +86 13789870769; Fax: +86 86057287 ;

E-mail: pingwsd@163.com
}

pepper noise, and it has improved fault-learning ability, particularly in extracting high-resolution images.

Using the multi-scale segmentation of multi-spectral aerial photography and comprehensive feature analysis tool, Mhangara et al. determined the threshold and most salient features for classification. The results indicate that the classification procedure generated accurate results [4]. Houwei used the object-oriented method to extract information from high-resolution aerial images. The results of this method are closer to the actual situation, and its accuracy is higher than the pixel-based method [5]. Considering the rich and detailed texture information of the Quickbird image, Tangwei built road extraction rules to extract important information of suburban roads. The classification accuracy was improved compared to the maximum likelihood method [6]. Xu Gaocheng used rich detailed information of the feature space and prominent texture information to build different rules to automatically extract different road information [7]. Based on multiscale segmentation, Huanliang used the nearest-neighbour classification method to extract road information. The result is improved because of the usage of spectral information, geometric and texture features of the images [8]. In this paper, considering the actual situation of the study areas, the object-oriented technology is used to automatically extract road information. There are two object-oriented methods in the following experiments: one is used in the segmentation scale and decision rules, and the other is based on multifeatures in experiments.

\section{DATA SOURCE AND PREPROCESSING}

In this paper, SPOT-5 and GaoFen-1 images are used to extract the road information. The SPOT-5 image shows the Feicheng mining area in Shandong Province on June $5^{\text {th }}$, 2011 [9]. There are wide and narrow roads in this study area. 


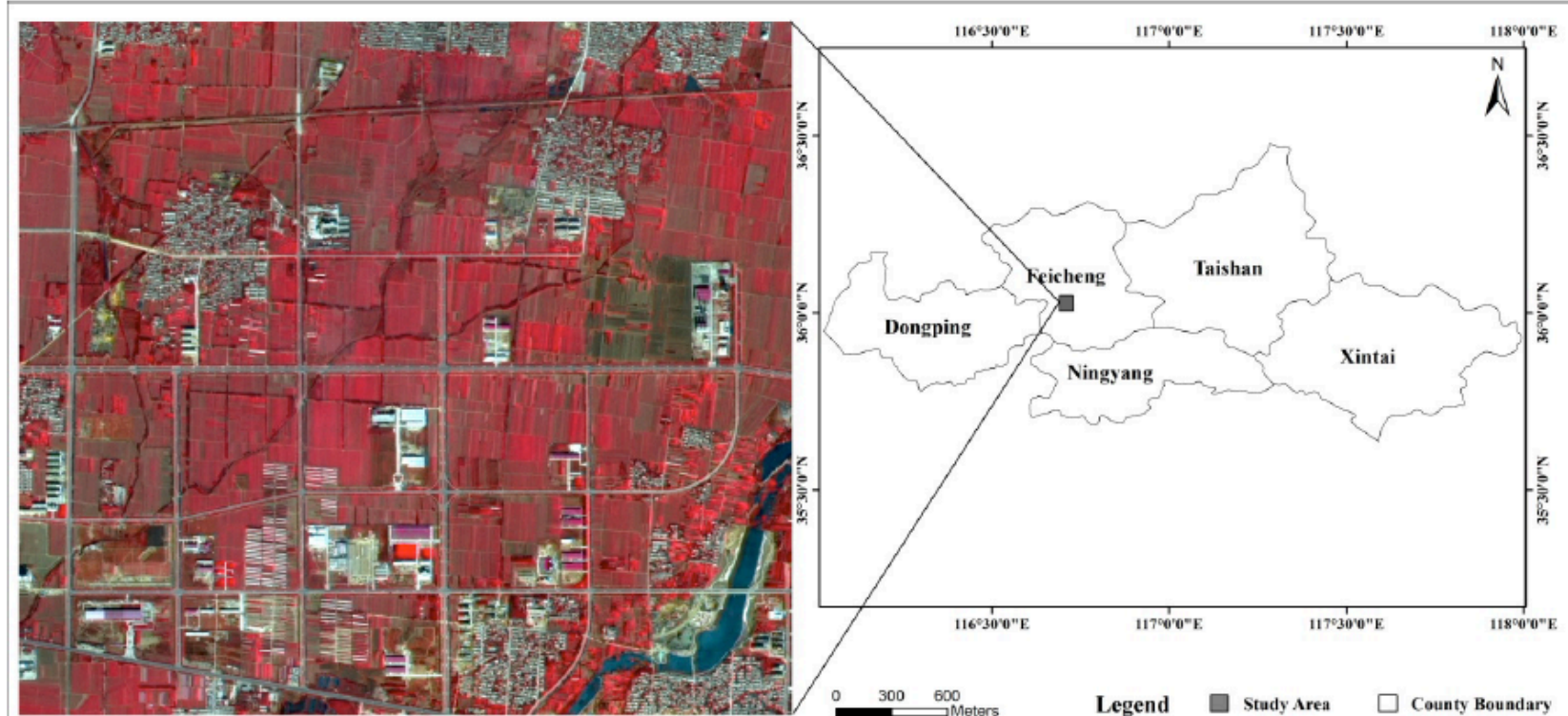

Fig. (1). Study area of feicheng mining area.

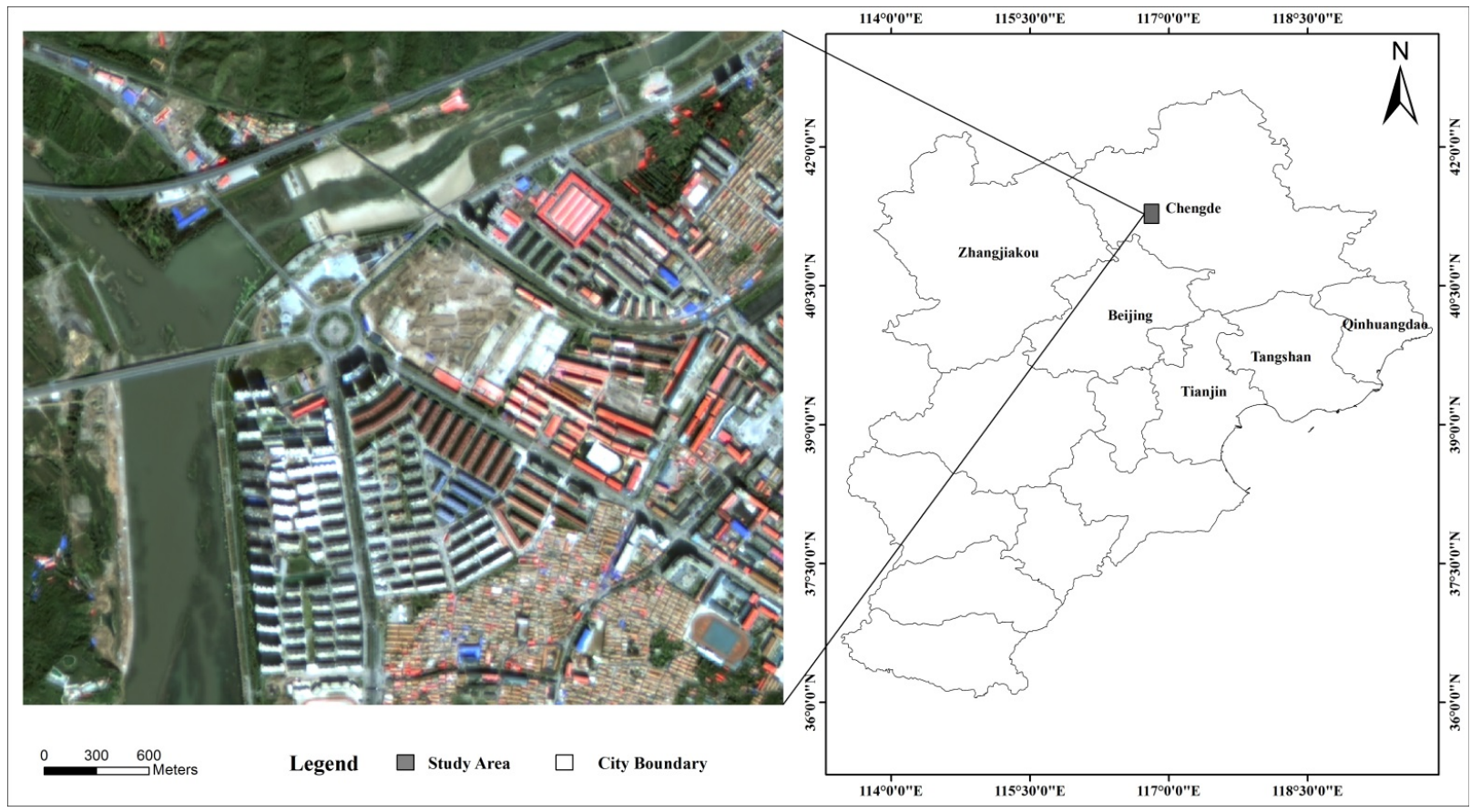

Fig. (2). Study area of the urban district.

Vegetation, buildings, water and other information in the image are also obvious, as shown in Fig. (1).

The GaoFen-1 image shows an area in Chengde City, Hebei Province on September 15 ${ }^{\text {th }}, 2013$. Compared with the SPOT-5 image, the road information is less obvious than other information, as shown in Fig. (2). The method based on segmentation scale-decision rules is used to extract road information in the SPOT-5 image, and the multi-featurebased method is used to extract road information in the Gaofen-1 image [10-12].

Image pre-processing mainly includes radiometric calibration, atmospheric correction, and geometric correction
[13]. The multi-spectral and panchromatic data images were fused based on Gram-Schmidt Pan sharpening to enhance the image. The images are displayed in false-colour and truecolour compositions.

\section{PRINCIPLE AND METHOD OF ROAD EXTRAC- TION}

Object-oriented methods collect neighbouring pixel information as the object to identify ground objects in the study areas. The objects domain offers more dimensions for image analysis and can take advantage of some geographic information system (GIS) analysis, as is shown in Fig. (3). 


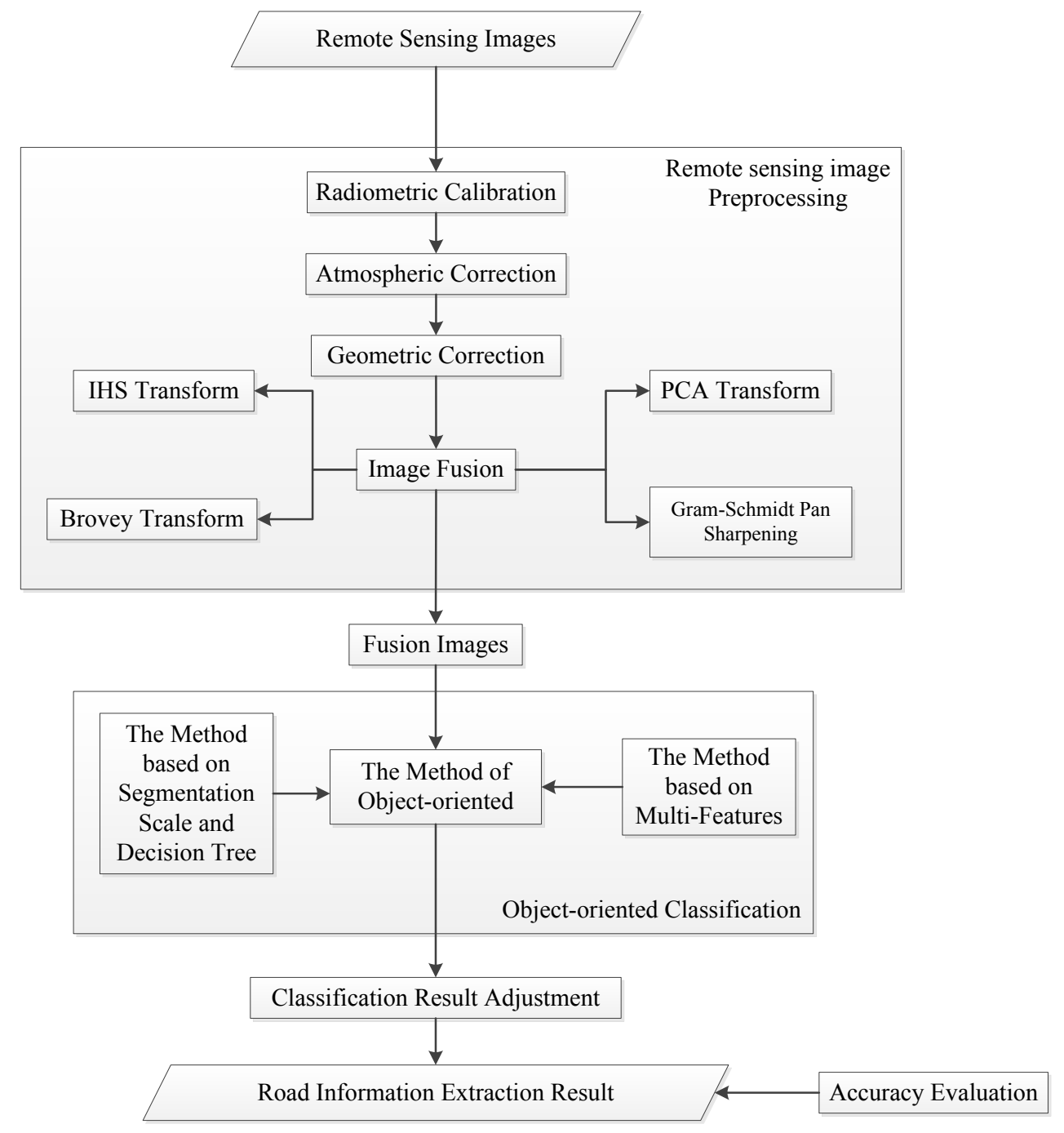

Fig. (3). Technology roadmap of the object-oriented method.

This method fully uses the space, texture and spectral information of a panchromatic and multi-spectral image to obtain a high-accuracy classification result. The classification procedure mainly includes object construction, object classification and accuracy evaluation [14].

Object construction refers to image segmentation. The multi-scale segmentation algorithm is the most widely used method. Considering the image spectral and shape features, the image spectral and shape heterogeneity comprehensive characteristic values of each band are calculated. Then, the weighted values are calculated according to the weight of each band [15]. The iteration is repeated until the weighted value of all segmented objects is greater than a specified threshold.

Object classification mainly includes supervised classification and rule-based classification. Object-oriented supervised classification differs from the traditional supervised classification based on pixels. There are more parameters in the sample selection and classification, which include the spectral, space and texture information. Rule classification is information extraction based on the properties and threshold value of the object [16-18].
The classification rules were built using the vegetation index, water body index, and values of each band in the method based on the segmentation scale-decision rules. Spectral features such as the intensity value, mean value, and standard deviation value of each band and geometric parameters such as the length-to-width ratio, compactness, and area are used in the multi-feature-based method.

There are many features to describe the characteristics of a road, which George Voss Elman has summarized as follows. The internal grey scale is homogeneous. It has larger grey contrast and edge gradient than neighbouring regions. The road is long with a limited curvature and smaller changes in width. Generally, roads are continuous and cross with one another in a network [19].

Although roads and buildings have different geometries, road and building information can be simultaneously extracted because of the similar spectral features. The extracted road information is significantly affected by buildings [20]. Hence, geometric features such as the length-width ratio, compactness, and density are used to extract the road information in addition to the spectral features. This paper uses 


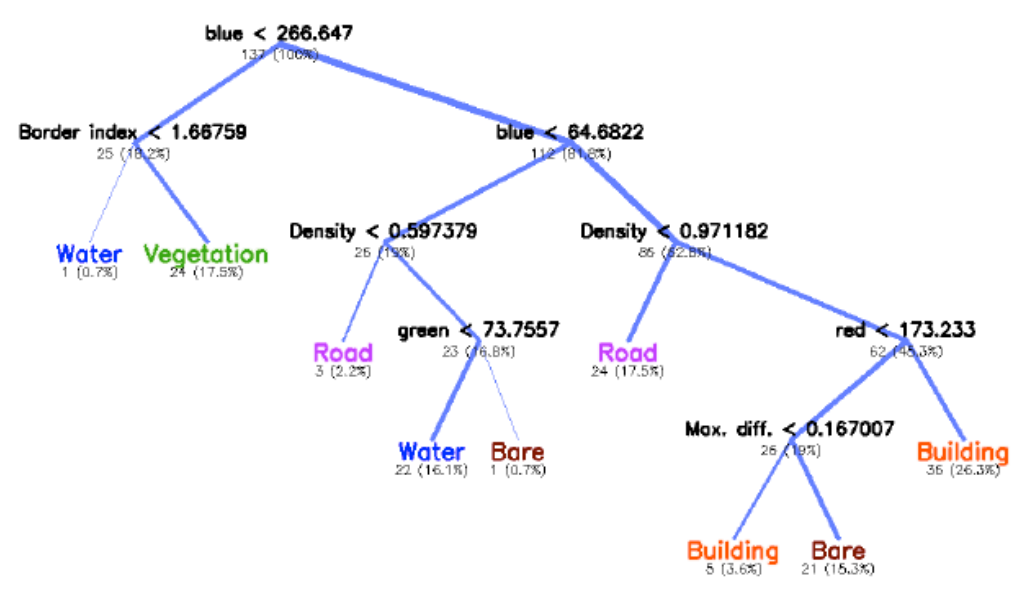

Fig. (4). Classification rules.

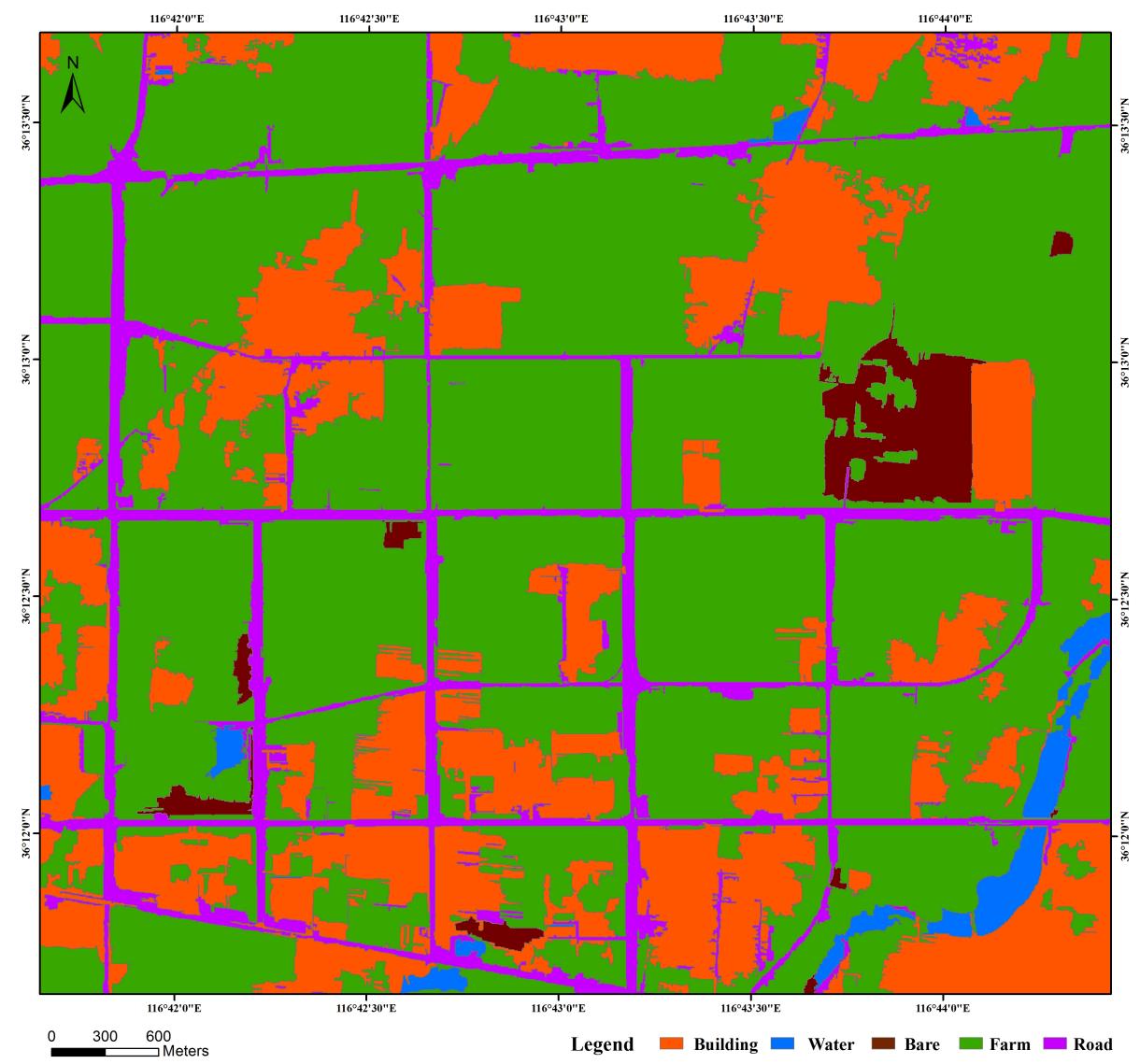

Fig. (5). Classification results of the method based on segmentation scale and decision tree.

the eCognition software to build road information extraction rules to automatically extract road information.

\section{ROAD INFORMATION EXTRACTION WITH DIF- FERENT METHODS}

\subsection{Method Based on Segmentation Scale and Decision} Tree

Because the SPOT-5 image has excellent spectral quality and rich texture information, the method based on the segmentation scale and decision tree is used in road information extraction. In this paper, we used the multi-scale segmentation technology to extract the road information; certainly repeated experiments are not finished until a reasonable segmentation scale is obtained. First, the samples are selected to describe the characteristics of typical features in the study area. Then, the classification rules are built based on these characteristics, which include the grey value of the band, density, etc. The specific rule of the parameters is in Fig. (4) [21-23].

The Border Index feature describes how jagged an image object is; the more jagged image the higher border index it has. $b_{v}$ is the image object border length; $l_{v}$ is the length of an image object $v ; w_{v}$ is the width of an image object $v$. 


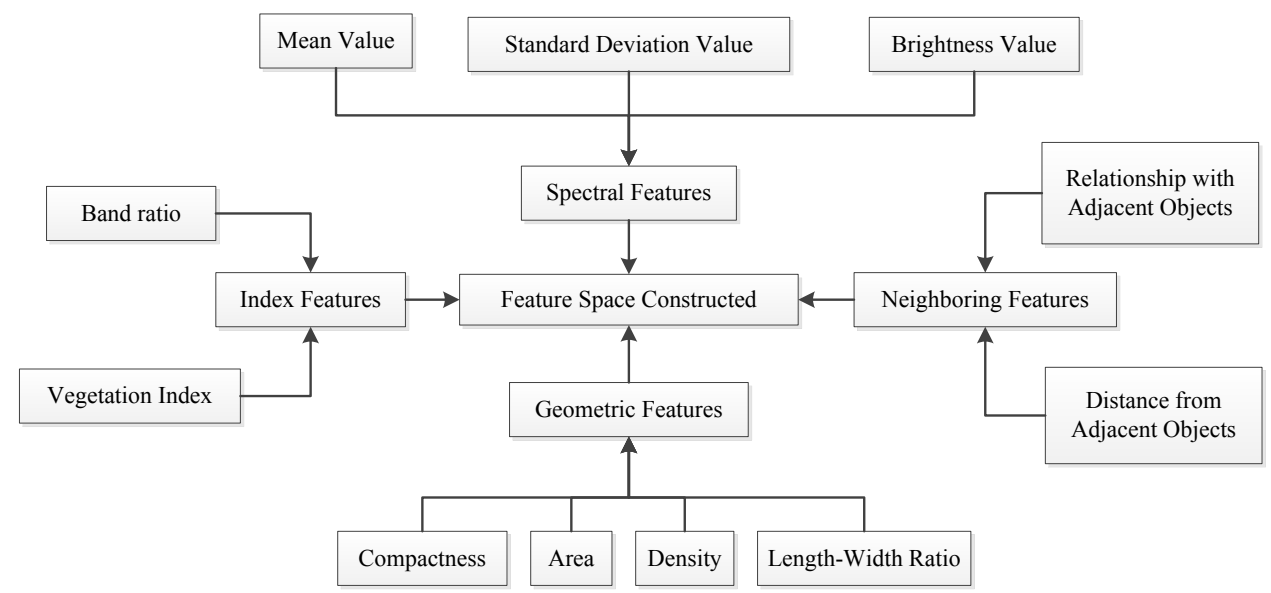

Fig. (6). Feature space constructed.

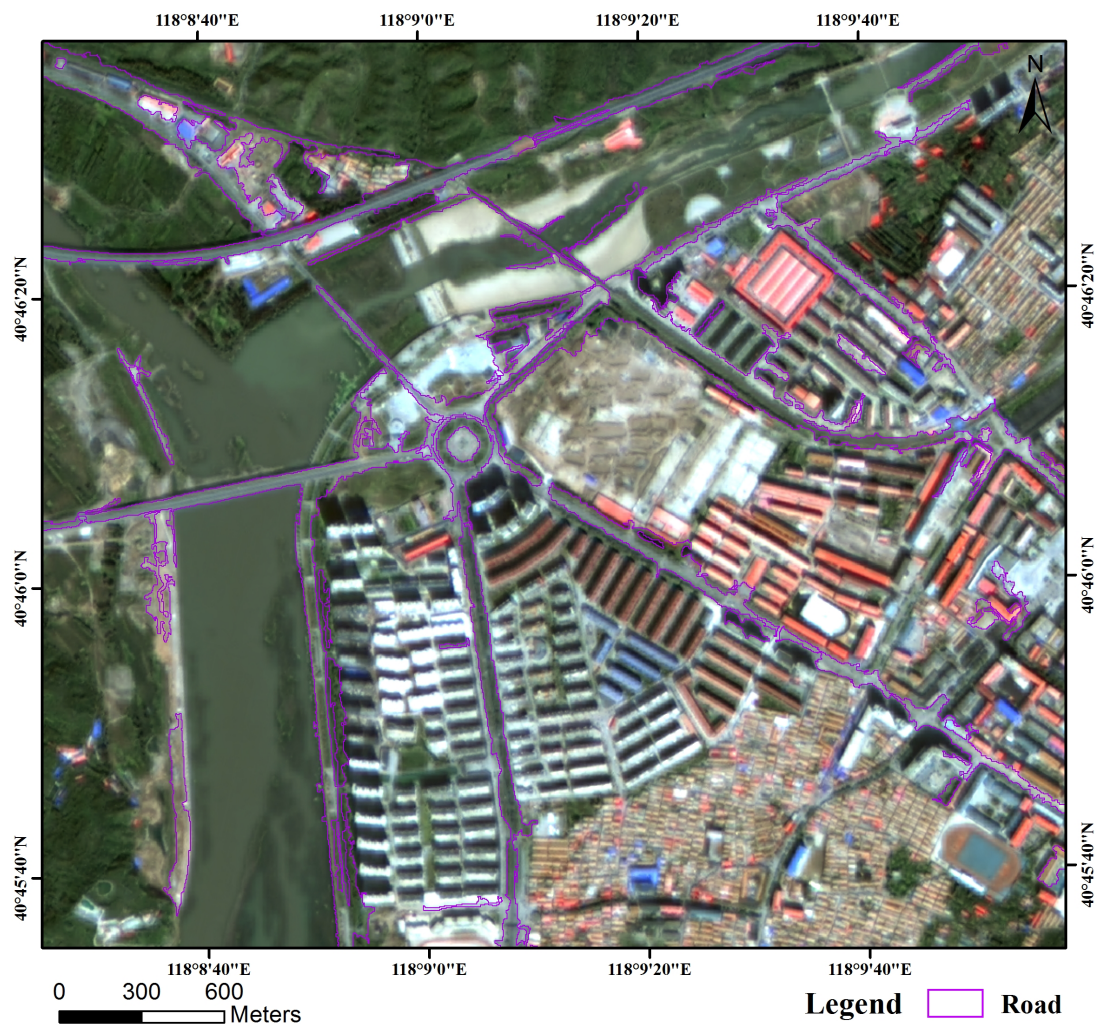

Fig. (7). Classification results of the multi-feature-based method.

Border Index $=\frac{b_{v}}{2\left(l_{v}+w_{v}\right)}$

The Density feature describes the spatial distribution of the pixels of an image object. $\sqrt{\# P_{v}}$ is the diameter of a square object with $\# P_{v}$ pixels; $\sqrt{\operatorname{Var} X+\operatorname{Var} Y}$ is the diameter of the ellipse.

Density $=\frac{\sqrt{\# P_{v}}}{1+\sqrt{\operatorname{Var} X+\operatorname{VarY}}}$

Max. diff is calculated as follows: I and $\mathrm{j}$ are image layers; $C(v)$ is the brightness of image object $\mathrm{v} ; \bar{C}_{i}(v)$ is the mean intensity of image layer $\mathrm{i}$ of image object $\mathrm{v} ; C_{j}(v)$ is the mean intensity of image layer $\mathrm{j}$ of image object $\mathrm{v} ; K_{B}$ are image layers with positive brightness weights.
$\operatorname{Max} . \operatorname{diff}=\frac{\max _{i, j \varepsilon K_{B}}\left|\bar{C}_{i}(v)-C_{j}(v)\right|}{\bar{C}(v)}$

The classification rules are used to extract information in the study area. A slight manual adjustment was performed to make the classification results more accurate. The road information is well extracted in excellent spectral quality and homogeneous grey value, as is shown in Fig. (5).

\subsection{Method Based on Multi-Features}

The method based on multi-features is used to extract the road information in the Gaofen-1 image. First, the feature space (Fig. 6) is constructed based on the spectral features, geometric features, neighbouring features and index features 


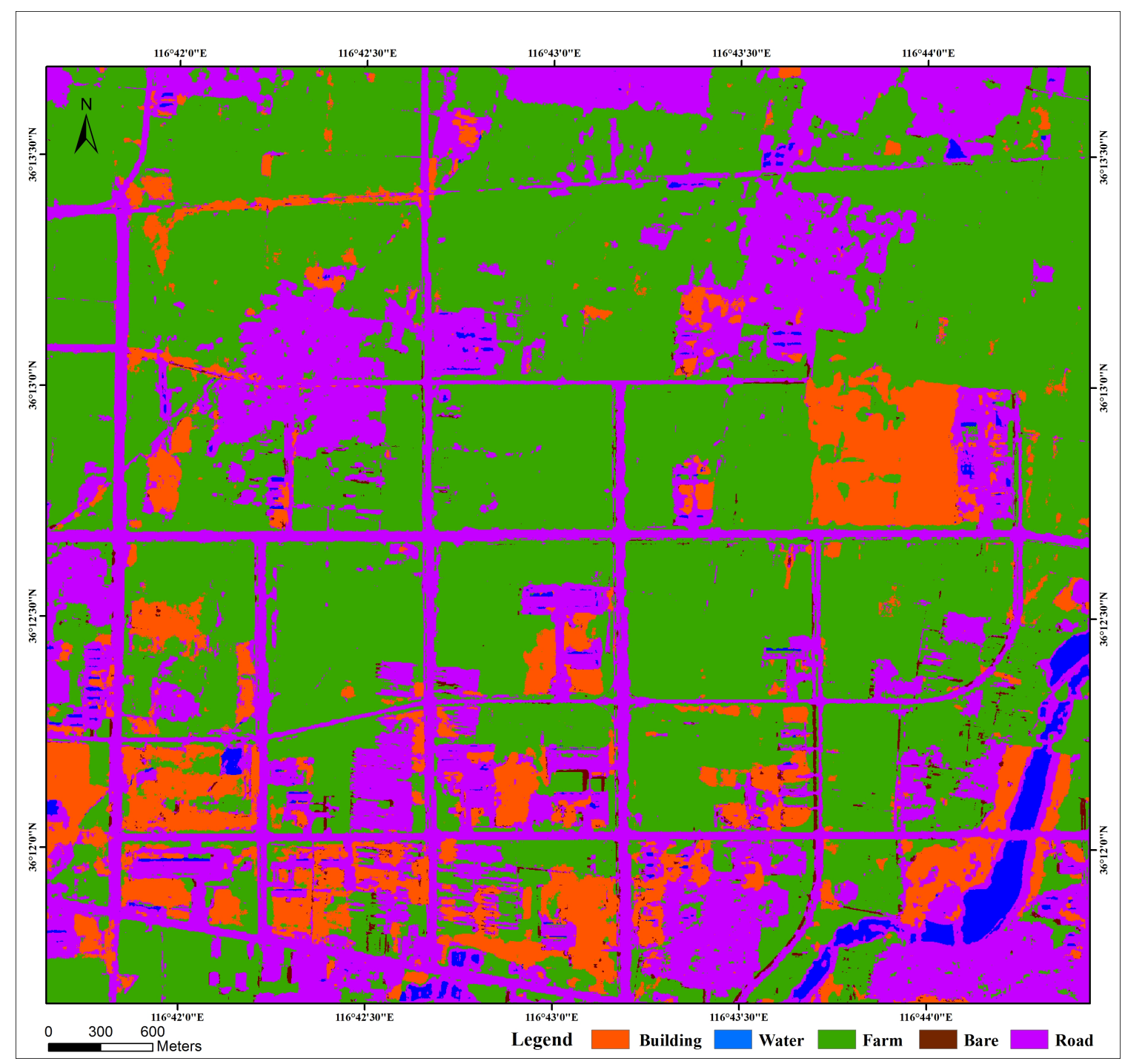

Fig. (8). Classification results of the supervised classification method.

of the image objects. To avoid data redundancy and unnecessary computation, the feature space is optimized using the eCognition software. Finally, these features are used to extract the road information.

The feature space that is constructed by the eCognition software cannot distinguish all ground objects. The values of the blue and near-infrared bands and the shape index are used to remove the building and water information from the road information.

Wide roads were extracted using this method (Fig. 7). However, the remaining problems must be solved in detail in subsequent studies.

\subsection{Comparison with the Traditional Supervised Classi- fication Method}

The supervised classification method was used to extract the road information in the SPOT-5 image. The pixel-based information extraction has the phenomenon of different spectra for one object, and different objects with identical spectra. Large numbers of buildings were considered roads (Fig. 8).
Comparing the results of the object-oriented and supervised classification methods, we can conclude that: (1) The object-oriented method extracts more continuous road information than the supervised classification method. (2) The former results are more consistent with the actual situation of the roads. Narrow-road information is also extracted. (3) The object-oriented method is more suitable for road information extraction of residential districts in mining areas.

The accuracy evaluation measures whether the classification results are credible. The common method is computing an Error Matrix [24-26]. The overall accuracy, user accuracy, production accuracy [27], Kappa coefficient and other accuracy statistics can be calculated from the error matrix. In the study area, sample points are selected and confirmed based on the existing thematic data. The error matrix is calculated as follows. Compared with the traditional method, the overall accuracy is $92.94 \%$, which is improved by $10 \%$, and Kappa coefficient is 0.9 (Table 1). Thus, the results are excellent $[28,29]$. 
Table 1. Error matrix from the object-oriented classification.

\begin{tabular}{|c|c|c|c|c|c|c|}
\hline & & \multicolumn{5}{|c|}{ Classification by indoor interpretation } \\
\hline & Building & Farm & Water & Road & Bare & Sum \\
\hline Building & 18 & & & & 1 & 19 \\
\hline Farm & 1 & 20 & & 3 & & 24 \\
\hline Water & & & 10 & & & 10 \\
\hline Road & 1 & 6 & & 27 & 4 & 28 \\
\hline Bare & & & & & & 4 \\
\hline Sum & 20 & 20 & 10 & 30 & 5 & \\
\hline \multicolumn{2}{|c|}{ Overall Accuracy } & $92.94 \%$ & & & \multicolumn{2}{|c|}{ Kappa Coefficient 0.9056} \\
\hline
\end{tabular}

\section{CONCLUSION}

Considering the image features and actual situation of the study areas, methods based on the segmentation scale, decision tree and multi-features are used to extract road information in this study. The road information is automatically extracted. Comparing with the traditional method, we can conclude that: (1) The object-oriented method can obtain better results, particularly in extracting the road information of mining areas and urban districts. (2) The road information that is extracted using the object-oriented method is more continuous. In addition, automatic or semi-automatic extraction is realized, which has high efficiency. (2) The road width that is extracted using the object-oriented method is more consistent with the actual situation. Narrow roads are also extracted. (3) The object-oriented method also has its limitation. The results depend on the segmentation quality, which implies that we need more time to perform many experiments to verify the appropriate segmentation scale. A method to determine the segmentation scale and combine effective feature parameters to achieve the best results must be further studied.

\section{CONFLICT OF INTEREST}

The authors confirm that this article content has no conflict of interest.

\section{ACKNOWLEDGEMENTS}

This study was supported by the Key Laboratory of Marine Surveying and Mapping in Universities of Shandong (Shandong University of Science and Technology) 2013B01 and by the study on iron and polymetallic mineral metallogenic conditions.

\section{REFERENCES}

[1] P.F. Wang, L. Wang, X.Z. Feng and P.F. Xiao. "Review of road extraction from remote sensing images", Remote Sensing Technology \& Application, vol. 24, no.3, pp. 284-290, 2009.

[2] K. Navulur, "Multispectral image analysis using the object-oriented paradigm", Crc Press, vol. 33, no. 1, pp. C24-C24, 2006.

[3] C.Y. Zhou, P. Wang, Z.Y. Zhang and C.T. Qi, "Classification of urban land based on object-oriented information extraction technology”, Remote Sensing Technology \& Application, vol. 23, no. 1, pp. 31-30, 2008
[4] P. Mhangara, J. Odindi, L. Kleyn and H. Remas. "Road extraction using object oriented classification", Visualisation Technical, pp. 45-50, 2011

[5] W. Hou, X.J. Lu, C. Zhang and J. Wang. "Object-oriented Information Extraction from High Resolution Imagery A Case Study for Recognition of Residential Area in Lixian County, Sichuan Province", Journal of Geo-Information Science, vol. 12, no. 1, pp. 119125, 2010.

[6] W. Tang, S. Zhao and P. Wang. "Object-oriented Road Information Extraction from High Resolution Imagery", Geo-Information Science, vol. 10, no. 2, pp. 257-262, 2008.

[7] G.C. Xu, J.T. Bi, X.X. Wang and H.J. Qu, "An experiment of automatic road extraction from high-resolution remote sensing image based on object-oriented technology", Remote Sensing Information, pp. 108-111, 2012.

[8] L. Huang, X.Q. Zuo, X.X. Zhang and Y. Liu, "Analysis of objectoriented road information recognition and extraction", Journal of Kunming University of Science \& Technology, vol. 35, no. 6, pp. 610,2010

[9] H.Yu, S.Q. Zhang, B. Kong and X F. Li, "Optimal segmentation scale selection for object-oriented remote sensing image classification", Journal of Image and Graphics, vol. 15, no. 2, pp.352-360, 2010.

[10] X.F. Li, S.Q. Zhang, F.W. Han, X.W. Qin and H.Yu, "Road extraction from high-resolution remote sensing images based on multiple information fusion", Acta Geodaetica et Cartographica Sinica, vol. 37, no. 2, pp. 178-184, 2008

[11] S.P. Chen and Y.S. Zhao, "Geo-Seienee Analysis of Remote Sensing", Surveying and Mapping Publishing Press, Beijing, 1990

[12] M. Polak, H. Zhang and M. Pi, "An evaluation metric for image segmentation of multiple objects", Image \& Vision Computing, vol 27, no. 8, pp. 1223-1227, 2009.

[13] A. Martin, H. Laanaya and A. Arnold-Bos, "Evaluation for uncertain image classification and segmentation", Pattern Recognition, vol. 39, no. 11, pp. 1987-1995, 2006.

[14] H.P. He, "Comparison of image fusion algorithms for IKONOS high spatial resolution satellite image", Science \& Technology Review, vol. 27, no. 5, pp.33-37, 2009.

[15] H. Murray, A. Lucieer and R. Williams, "Texture-based classification of sub-Antarctic vegetation communities on Heard Island", International Journal of Applied Earth Observation \& Geoinformation, vol. 12 , no. 3, pp. 138-149, 2010.

[16] G.J. Hay and D.J. Marceau, "Multiscale object-specific analysis (MOSA): An Integrative Approach for Multiscale Landscape Analysis", Remote Sensing Image Analysis, pp. 71-92, 2004.

[17] M. Kim, T.A. Warner, M. Madden and D.S. Atkinson. "Multi-scale GEOBIA with very high spatial resolution digital aerial imagery: scale, texture and image objects", International Journal of Remote Sensing, vol. 32, no. 10, pp. 2825-2850, 2011.

[18] Y.H. Chen, T. Feng, P.J. Shi and J.F. Wang, "Classification of remote sensing image based on object oriented and class rules", Geomatics and Information Science of Wuhan University, vol. 31, no. 4, pp. 316-320, 2006. 
[19] J. Wang, S. L. Lu, B. F. Wu, N. N. Yan and L. Pei," Land Cover Change in Baiyangdian Wetland", Journal of Geo-Information Science, vol. 12, no. 2, pp. 292-300, 2010

[20] R. Nevatia and K. R. Babu. "Linear feature extraction and description", Computer Graphics \& Image Processing, vol. 13, no. 3, pp. 257-269, 1980.

[21] F. Wang and R. Newkirk, "A knowledge-based system for highway network Extraction", IEEE Transactions on Geoscience \& Remote Sensing, vol. 26, no. 5, pp. 525-531, 1988.

[22] H. D. Cheng, X. H. Jiang, Y. Sun and J. Wang, "Color image segmentation: advances and prospects", Pattern Recognition, vol. 34, no. 12, pp. 2259-2281, 2001.

[23] N. R. Pal and S. K. Pal. "A review on image segmentation techniques", Pattern Recognition, vol. 26, no. 39, pp. 1277-1294,1993.

[24] P. Sahoo, C. Wilkins and J. Yeager, "Threshold selection using Renyi's entropy", Pattern Recognition, vol. 30, no. 1, pp. 71-84. 1997.
[25] R. G. Congalton, "A review of assessing the accuracy of classifications of remotely sensed data", Remote Sensing of Environment, vol. 17 , no. 1, pp. 35-46,1991.

[26] J. A. Richards, "Classifier performance and map accuracy", Remote Sensing of Environment, vol. 57, no. 3, pp.161-166, 1996.

[27] S. V. Stehman, "Selecting and interpreting measures of thematic classification accuracy", Remote Sensing of Environment, vol. 62, no. 1, pp. 77-89, 1997.

[28] M. Story and R. G. Congalton, "Accuracy assessment: a user's perspective", Photogrammetric Engineering \& Remote Sensing, vol. 52, no. 3, pp. 397-399, 1986.

[29] L. L. F. Janssen and F. J. M. Wel, "Accuracy assessment of satellite derived land-cover data: A review", Photogrammetric Engineering \& Remote Sensing, vol. 60, no. 4, pp. 419-426, 1944.

Received: May 26, 2015

Revised: July 14, 2015

Accepted: August 10,2015

(C) Ping et al.; Licensee Bentham Open

This is an open access articles licensed under the terms of the Creative Commons Attribution-Non-Commercial 4.0 International Public License (CC BY-NC 4.0) (https://creativecommons.org/licenses/by-nc/4.0/legalcode), which permits unrestricted, non-commercial use, distribution and reproduction in any medium, provided that the work is properly cited. 